\title{
DISCORDANT EPILEPSY IN MONOZYGOUS TWINS
}

Twelve monozygotic twins, discordant for epilepsy, were analysed for nonhereditary etiological factors by clinical history, MRI, and quantitative brain volume studies at the Brain Research Institute, University of Melbourne, Victoria, Australia. A major acquired factor explained discordance in 4 of 12 affected twins. All had temporal lobe epilepsy, 2 twins had a prolonged febrile convulsion in early childhood and hippocampal sclerosis on MRI, and 2 had a pre- or perinatal ischemic-hypoxic insult with ischemic lesions. MRI showed significant cortical maldevelopment (MCD) in 4 of the remaining 8 affected MZ twins without major acquired risk factors. In the remaining 4 twin pairs with discordant seizures unexplained by major acquired factors or MCDs, 2 twin pairs had differences in quantitative MRI brain volumes, indicative of subtle maldevelopment and a nonhereditary etiology. Both affected twins had idiopathic generalized epilepsy and larger hippocampal and intracranial volumes than the unaffected co-twins. No reason for discordance was uncovered in 2 or 12 twins. (Briellmann RS, Jackson GD, Torn-Broers Y, Berkovic SF. Causes of epilepsies: insights from discordant monozygous twins. Ann Neurol January 2001;49:45-52). (Respond: Dr Jackson, Brain Research Institute, Neurosciences Building, Repatriation Campus, West Heidelberg, 3081, Victoria, Australia).

COMMENT. Discordance for epilepsy in monozygous twin pairs is explained by noninherited factors. These include major acquired clinical risk factors, malformation of cortical development demonstrated by MRI, and quantitative brain volume changes. Idiopathic generalized epilepsy, usually considered primarily genetic in etiology, may be caused by underlying subtle developmental abnormalities. Occult acquired factors may explain cases of cryptogenic epilepsy.

\section{IDIOPATHIC GENERALIZED EPILEPSY WITH HEMICONVULSIONS}

Three adolescent patients with hemiconvulsive seizures and 3-cps generalized spike-and-wave discharges on EEG are reported from the National Hospital, Queen Square, London, UK. All are seizure-free following treatment with sodium valproate or/and lamotrigine, whereas prior treatment with carbamazepine was only partially effective. The diagnosis of idiopathic generalized epilepsy is supported by the EEG, normal CT or/and MRI, and pharmacological response. (Kiley MA, Smith SJM, Sander JW. Idiopathic generalized epilepsy presenting with hemiconvulsive seizures. Epilepsia Dec 2000;41:1633-1636). (Reprints: Dr SJM Smith, National Hospital of Neurology and Neurosurgery, Queen Square, London WC1N 3BG, UK).

COMMENT. Idiopathic generalized epilepsy (IGE) is a heterogeneous syndrome. Asymmetrical cortical hyperexcitability is favored as the explanation for the hemiconvulsive seizures in the above small series. In the monozygous discordant twin study (Briellmann et al, 2001), IGE was associated with asymmetrical brain volume abnormalities on quantitative MRI, suggesting a subtle maldevelopment that might explain hemiconvulsive seizures in rare cases.

\section{PREDICTION OF COMPLIANCE WITH EPILEPSY TREATMENT}

Sociocultural, medical, family environment, and cognitive factors predictive of adherence to treatment of epilepsy were analysed in 119 children, age 4 to 14 years, enrolled in a longitudinal study at the first visit to the seizure clinic, Los Angeles Children's Hospital, CA. A foreign language, most commonly Spanish, was spoken in the home in $45 \%$ of families. Families with less parental education, non-English speaking recent immigrants with lower incomes and 
highly stressful lives, were more likely to keep appointments and to comply with treatment schedules than those with cultural and socioeconomic advantages. Cognitive functioning (IQ) was positively correlated with adequate drug levels. Seizure frequency and severity were not correlated with treatment adherence. Behavioral comorbidity was associated with poor adherence to medication and attendance. Parental report of medication adherence was minimally correlated with adherence based on drug levels. Adherence measures were statistically independent of each other, emphasizing the need to use multiple predictive factors. (Mitchell WG, Scheier LM, Baker SA. Adherence to treatment in children with epilepsy: who follows "doctor's orders?" Epilepsia Dec 2000;41:1616-1625). (Respond: Dr Wendy Mitchell, Neurology Division, Children's Hospital Los Angeles, 4650 Sunset Blvd, Los Angeles, CA 90027).

COMMENT. Contrary to expectations, the most striking positive measure of adherence to therapy and attendance of patients with epilepsy in this study was an acculturative risk factor among non-English speaking recent immigrants with lower incomes and highly stressful lives. Seizure severity was not associated with adherence outcome. One factor not addressed in this study was the physician's fluency with foreign language or need for clinic interpreters that might detract from patient communication. In my early years at Children's Memorial Hospital, Chicago, in the 1960's, one of my fellows in training, Dr Winston Ortiz, later of Ponce, Puerto Rico, was adept at obtaining compliance with epilepsy treatment from Spanish-speaking parents at our Seizure Clinic. His clinical acumen, fluency with the language, and gentle but commanding persuasion engendered trust and faithful adherence to treatment regimens and appointment schedules. The rapport between physician and patient is perhaps the most important predictive factor of compliance and success of epilepsy management. In recent years we physicians have also become dependent on the support and expertise of nurse practitioners who strengthen the physician-patient relationship and contribute to treatment compliance and effectiveness.

\section{BRAIN MALFORMATIONS}

\section{CALLOSAL AGENESIS AND INTERHEMISPHERIC CYSTS}

Imaging studies of 25 cases of agenesis of the corpus callosum with interhemispheric cyst were retrospectively reviewed at the University of California, San Francisco, and Harvard Medical School, Boston. A classification based on morphology was developed from the study of CTs of 6 patients and MiRIs of 19 patients. Two major types are recognized, each with subtypes: Type 1 cysts formed from extension or diverticulation of the third or lateral ventricles; and Type 2 loculated cysts not communicating with ventricles.

Type 1 cyst subtypes are characterized as follows: 1a) associated with communicating hydrocephalus; 1b) obstructive hydrocephalus caused by diencephalic malformation and blockage of fluid from 3 rd ventricle to aqueduct; 1c) associated with hemisphere hypoplasia and microcephaly.

Type 2 cyst subtypes: 2a) multiloculated cysts associated only with callosal agenesis; 2b) associated with deficiency of falx, heterotopia, polymicrogyria, and Aicardi syndrome; 2c) associated with subcortical heterotopia; 2d) interhemispheric arachnoid cysts. The majority of patients were male, except those with $2 \mathrm{~b}$ cysts. (Berkovich AJ, Simon EM, Walsh CA. Callosal agenesis with cyst. A better understanding and new classification. Neurology January ( 2 of 2) 2001;56:220-227). (Reprints: Dr A James Berkovich, Department of Neuroradiology, L371, University of California San Francisco, 505 Parnassus Ave, San Francisco, CA 94143). 